Results A total of 49 urine specimens, 50 vaginal and 33 endocervical swabs were evaluated. Positive percent agreement was 92.0 for urine, and $100 \%$, for both of the swab specimen types compared to the routine assay. Negative percent agreement between the two assays was $100 \%$ for all three specimen types. Kappa scores between the two assays were $0.918,1.000$, and 1.000 for urine, vaginal, and endocervical swabs, respectively.

Conclusions The TV LDT assay, performed on the Abbott $m 2000$ platform, has excellent agreement with a molecular assay for TV being used in our laboratory. Advantages to using the m2000 for TV testing include automation and the use of residual DNA from the CT/NG assay for TV detection.

\section{P5.093 EVALUATION OF A NEW AMPLIFIED DNA ASSAY ON THE BECTON DICKINSON VIPER SYSTEM IN EXTRACTED MODE FOR THE DETECTION OF TRICHOMONAS VAGINALIS FROM VAGINAL SPECIMENS}

doi:10.1136/sextrans-2013-051184.1137

\begin{abstract}
1.2B Van Der Pol, 1J A Williams, 'L Eddleman, 1,3D Fuller, ${ }^{4}$ S Taylor, ${ }^{5} \mathrm{~J}$ Schwebke, ${ }^{6} \mathrm{~J}$ Lebed, ${ }^{7} \mathrm{~B}$ Smith, ${ }^{8} \mathrm{M}$ Nye, ${ }^{9} \mathrm{C}$ Gaydos. 'Indiana University School of Medicine, Indianapolis, IN, United States; 'Indiana University School of Public Health, Bloomington, IN, United States; ${ }^{3}$ Wishard Health Services, Indianapolis, IN, United States; ${ }^{4}$ Louisiana State University Health Sciences Center, New Orleans, LA, United States; ${ }^{5}$ University

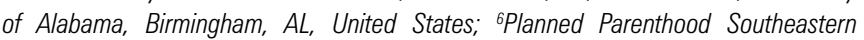
Pennsylvania, Philadelphia, PA, United States; 'Planned Parenthood of Gulf Coast Inc., Houston, TX, United States; ${ }^{8}$ LabCorp, Burlington, NC, United States; ${ }^{9} T$ The Johns Hopkins University, Baltimore, MD, United States
\end{abstract}

Background The BD Probe Tec ${ }^{\mathrm{TM}}$ Trichomonas vaginalis (TV) $\mathrm{Q}^{\mathrm{x}}$ amplified DNA Assay (TVQ) is a new test for qualitative detection of TV DNA that can be performed on the automated BD Viper System. The objective of this study was to compare the performance of this new assay to a patient infected status (PIS) and to an FDA approved molecular assay using vaginal swabs.

Methods Vaginal swabs were obtained from women attending STD or family planning clinics at 7 sites. A patient collected vaginal swab was tested by TVQ; APTIMA TV (ATV) testing was performed using a clinician obtained vaginal swab according to the package insert. Additional clinician obtained vaginal swabs were used for wet mount and culture. A patient was considered infected if either the wet mount or culture was positive for TV and not infected if both tests were negative. Agreement between the TVO and ATV assays was assessed using Kappa statistics.

Results Data were available for TVQ evaluation from 838 women, 116 of whom were defined as infected with TV. Despite being in the definition of the PIS, wet mount still had a sensitivity of only $68.7 \%$ which was statistically lower than the other assays $(p<0.001)$. TVQ sensitivity and specificity estimated based on the PIS were $94.2 \%$ and $99.7 \%$, respectively. TVQ performed similarly to the ATV assay $(\kappa=0.938)$.

Conclusions The TVQ assay performed significantly better than wet mount and had comparable sensitivity and specificity to an FDA approved molecular assay for the detection of TV. This study provides additional evidence of the poor performance of wet mount for TV. The use of patient collected vaginal swabs for the detection of TV DNA provides clinicians with the opportunity to increase efficiency within the clinic while obtaining improved results over wet mount.

\section{P5.094 EVALUATION OF GENTAMICIN SUSCEPTIBILITY OF NEISSERIA GONORRHOEAE ISOLATES IN ARGENTINA}

doi:10.1136/sextrans-2013-051184.1138

R Gianecini, I Pagano, C Oviedo, S Reggiane, M Vacchino, Gonococcal Antimicrobial Susceptibility Surveillance Argentinean Network, P G Galarza. National Institute of Infectious Diseases, Buenos Aires, Argentina
Introduction The effective treatment of infection by Neisseria gonorrhoeae $(\mathrm{Ng})$ is critical for the individual patient management and essential in the control of gonorrhoea. The emergence of decreased susceptibility to third generation cephalosporins and its association with treatment failure in many regions of the world can quickly make them unsuitable as first-line therapy. Thus it becomes necessary to consider alternatives for future therapeutics. The aminoglycoside gentamicin, was chosen as an alternative treatment after the emergence of penicillinase-producers strains in Africa. This responds to its low cost, also due to the fact that it can be administered in a single dose of $240 \mathrm{mg}$ and because studies showed cure rates of $>95 \%$. Despite some treatment failure reports, gentamicin has proven successful in the treatment of gonococcal urethritis for many years. In Argentina, no susceptibility data are available.

Materials and Methods: Retrospective study of a total of 355 $\mathrm{Ng}$ isolates derived to our laboratory for susceptibility studies in 2011 from 13 provinces. MIC to gentamicin was determined by agar dilution method according to CLSI. We used Ng ATCC 49226 as control for dilutions of antibiotics, using the interpretation criteria reported in bibliography.

Results Gentamicin susceptibility showed that $99.7 \%$ of Argentine isolates were in a narrow range of MIC $(4-8 \mu \mathrm{g} / \mathrm{ml})$ with $74.6 \%$ showing an MIC of $8 \mu \mathrm{g} / \mathrm{ml}$. The MIC range was $4-16 \mu \mathrm{g} / \mathrm{ml}$, MIC 50 and MIC 90 agreed $8 \mu \mathrm{g} / \mathrm{ml}$. A 74.6\% (265/355) isolates included in this study showed resistance to one or more of the following antibiotics: penicillin (36.3\%), tetracycline (43.9\%) and ciprofloxacin (48.4\%). Conclusions The Argentine gonococcal population susceptibility to gentamicin is similar to that reported by other regions of the world. In vitro studies of regular assessment would be needed to ensure the effectiveness of gentamicin as alternative drug for the treatment of gonorrhoea.

\section{P5.095 CEFIXIME TREATMENT FAILURE IN INFECTIONS WITH CEFIXIME SUSCEPTIBLE N. GONORRHOEA STRAINS}

doi:10.1136/sextrans-2013-051184.1139

B Kranzelbinder, M Wiednig, W Aberer, P Komericki. Department of Dermatology and Venereology, Medical University of Graz, Graz, Austria

Background In the last years the Gram-negative bacterium Neisseria gonorrhoeae, already known to be resistant to penicillins, tetracyclines, macrolides and fluoroquinolones has raised attention by developing resistance and consequently treatment failures in some cases to the recommended first line treatment: extended-spectrum cephalosporins (ceftriaxone and cefixime). Therefore bacterial culture, the gold standard for definite diagnosis should be performed for antibiotic susceptibility testing, beside the widely used nucleic acid amplification testing (NAAT). However we could observe discrepancies between cefixime susceptible N. gonorrhoeae cultures and clinical treatment failures for some years.

Methods In this retrospective study, 2006-2012, clinical outcome data of patients with acute gonococcal urethritis/cervicitis, oral cefixime treatment $(400 \mathrm{mg}$, one dose) and cefixime susceptible $\mathrm{N}$. gonorrhoeae culture were collected at the STD outpatient clinic of the Department of Dermatology and Venereology, Medical University of Graz, Austria. The diagnosis was made by microscopy (Gram or methylene blue staining), culture including antimicrobial susceptibility testing and in situ hybridization (GenProbe Pace II) of urethral/cervical swab specimens. Control urethral/cervical swaps were performed within one to two weeks.

Results Out of total 218 patients with gonorrhoea, 120 patients fulfilled the inclusion criteria. 14 of 120 (11.7\%) showed a treatment failure after oral cefixime despite a positive susceptibity testing. Most treatment failures were observed in 2011 (3/11; 21.4\%) and 2012 (4/17; 19\%). Rates for 2007, 2008 and 2009 were 2/12; 14.3\%, $3 / 16 ; 15.8 \%$ and $2 / 11 ; 15.4 \%$. In 2006 and 2010 , no treatment failure in cefixime susceptible $\mathrm{N}$. gonorrhoeae infections was seen. 
Conclusion Culture and antibiotic susceptibility testing remains essential in gonorrhoea management in regard of recent surveillance data suggesting that cephalosporins are becoming less effective in the treatment of gonorrhoea. Our data suggest that treatment failures with oral cefixime may occur even in infections with cefixime susceptible $\mathrm{N}$. gonorrhoeae strains.

\section{P5.096 ESTIMATING THE POTENTIAL ECONOMIC IMPACT OF ANTIMICROBIAL RESISTANCE IN NEISSERIA GONORRHOEAE IN THE UNITED STATES}

doi:10.1136/sextrans-2013-051184.1140

H Chesson, K Owusu-Edusei, Jr, R D Kirkcaldy, T L Gift, H S Weinstock. Centers for Disease Control and Prevention, Atlanta, GA, United States

Background Antimicrobial resistance to treatment can hinder gonorrhoea prevention and control efforts, thereby leading to increases in gonorrhoea incidence. We estimated the economic burden of potential increases in gonorrhoea incidence in the US as a result of emerging cephalosporin resistance.

Methods The potential increase in gonorrhoea due to resistance was based on an analysis of historical gonorrhoea incidence and ciprofloxacin resistance data. We used clinic-level resistance data from the Gonococcal Isolates Surveillance Project (GISP) and city-level gonorrhoea incidence rates from surveillance data for 17 GISP cities from 1991 to 2006. We performed a regression analysis in which the gonorrhoea rate (log) was the dependent variable and the independent variable of interest was the percentage of GISP isolates (from the clinic in the respective city) resistant to ciprofloxacin. To estimate the cost of potential increases in gonorrhoea, we used STICFigure, a spreadsheet programme that applies published equations of the economic impact of STDs.

Results The regression analysis found a significant, positive association $(p<0.01)$ between ciprofloxacin resistance and gonorrhoea incidence at the city level. The results suggested that an increase in resistance from $0 \%$ to $10 \%$ of isolates could result in a net increase in gonorrhoea of about $7 \%$ (range: $3 \%-12 \%$ ) in the first year and 17\% (range: 6\% - 28\%) after ten years. Over ten years, the estimated impact would be substantial: 48,000 additional cases of PID, 5,000 additional cases of epididymitis, and 560 additional HIV infections, with direct medical costs totaling $\$ 405$ million (range: $\$ 152$ million - \$689 million).

Conclusions Ciprofloxacin resistance was associated with increased gonorrhoea rates, despite availability of alternative treatments at the time. Correspondingly, emerging cephalosporin resistance could have substantial health and economic consequences. Efforts to control the spread and reduce the consequences of resistant strains can mitigate this potential burden.

\section{P5.097 POTENTIAL POPULATION-LEVEL IMPACT OF REPLACING ORAL GONOCOCCAL THERAPY WITH INTRAMUSCULAR THERAPY}

doi:10.1136/sextrans-2013-051184.1141

M R Golden, L Barbee, R Kerani, J Dombrowski. University of Washington, Public Health - Seattle \& King County, Seattle, WA, United States

Background The role of oral drugs in gonorrhoea treatment is controversial, and the effect of eliminating oral treatments is unknown. Methods We created an arithmetic model to estimate the number of treatment failures (including no treatment) among persons with gonorrhoea and their first generation sex partners comparing current treatment patterns to treatment using only intramuscular drugs. Our base case scenario assumed: decreased susceptibility in $2 \%$ and $5 \%$ of cases in heterosexuals and men who have sex with men (MSM), respectively; oral therapy use in $30 \%$ of heterosexuals and $15 \%$ of MSM; treatment failure in $10 \%$ of persons with decreased susceptibility gonorrhoea given oral therapy; elimination of oral therapy results in $5 \%$ of oral treatment patients going untreated; expedited partner therapy (EPT) is offered to $40 \%$ of heterosexuals and increases the number of infected partners treated per case by 0.165 .

Results In our base case scenario, elimination of oral treatment decreased the number of decreased susceptibility treatment failures relative to the total number of decreased susceptibility cases by $0.8 \%$ if one ignores the effect of EPT, and increased decreased susceptibility cases by $0.8 \%$ with an EPT effect included. Total gonorrhoea cases increased by $0.8 \%$ and $4 \%$, with and without an EPT effect, respectively. Assuming 50\% of heterosexuals and $25 \%$ of MSM receive oral therapy at baseline and that $20 \%$ of decreased susceptibility cases fail oral treatment, elimination of oral therapy diminished decreased susceptibility cases $2.6 \%$ and $1.4 \%$ with and without an EPT effect, respectively, while increasing total gonorrhoea cases $1.5-4.8 \%$.

Conclusions Given plausible current levels of treatment efficacy, eliminating oral gonorrhoea therapy in the U.S. would likely have a small effect on decreased susceptibility treatment failures, and would somewhat increase gonorrhoea morbidity. These findings do no incorporate longer-term transmission effects, but highlight the importance of developing effective oral gonorrhoea treatment options.

\section{P5.098 CLINICAL PREDICTION OF FLUOROQUINOLONE SUSCEPTIBILITY, DIRECTLY FROM RESIDUAL ROUTINE NAAT GONOCOCCAL-POSITIVE SAMPLES USING A GYRA SNP DETECTION ASSAY}

doi:10.1136/sextrans-2013-051184.1142

'V F Miari, 'M J Pond, ' $\mathrm{K}$ Laing, ' $\mathrm{H}$ Jagatia, 1 I Monahan, 1,2T Planche, ${ }^{1} \mathrm{P}$ D Butcher, ${ }^{1,3} \mathrm{~S}$ T Sadiq. 'St George's University of London, London, UK; '2St George's Healthcare NHS Trust, Department of Medical Microbiology, London, UK; ${ }^{3}$ Department of Genitourinary Medicine, St George's Healthcare NHS Trust, London, UK

Background Antimicrobial resistance in Neisseria gonorrhoeae (NG) poses significant challenges for empirical therapy, heightened by nucleic acid amplification tests (NAAT) use over culture for diagnosis. Molecular susceptibility testing, directly on NAAT-positive clinical samples may allow fluoroquinolone use, currently precluded because community resistance rates are $>5 \%$. We determined whether a genotypic resistance test performed directly on NG NAAT positive routine clinical samples predicted susceptibility to ciprofloxacin with $>95 \%$ confidence.

Methods A real time PCR SNP detection assay targeting the C > T SNP in codon 91 (S91P) of gyrA, commonly linked with other gyrA resistance associated SNPs, was used on 81 previously identified NG isolates, tested for ciprofloxacin MICs by E-test after sample blinding (Phase 1) and also (Phase 2) on 103 blinded clinical samples from 89 patients, positive by NAAT and culture ( 25 women, 64 men; 21 cervical, 3 vaginal, 1 urethral, 48 urine, 10 throat and 21 rectal).

Results Phase 1 and Phase 2: 61/81(75\%) and 68/103(66\%) respectively were phenotypically susceptible to ciprofloxacin; $81 / 81$ and $87 / 103$ respectively of S91P assays worked. Phase 2 assay failure was not associated with sample site. Of Phase 2 assays that worked, predictive values for identifying ciprofloxacin susceptible gonorrhoea, using wild-type (S91P absence), and resistant gonorrhoea, using S91P presence, on clinical samples from: genital sites was 100\%(95\% CI: 91\%-100\%)/86\%(67\%-95\%) respectively; non-genital sites was $93 \%(68 \%-99 \%) / 92 \%(65 \%-99 \%)$ respectively; overall was $98 \%(90 \%-100 \%) / 88 \%(73 \%-95 \%)$ respectively. Among all 89 patients, assay use would have identified $47(53 \%)$ as treatable with ciprofloxacin, one incorrectly (predictive value of assay for susceptibility $97.9 \%(88.9 \%-99.6 \%)$. However, nearly $80 \%$ of men and $60 \%$ of women received treatment for gonorrhoea before these results would have been available. 